\title{
Influence of Carbon Nanostructure and Oxygen Moieties on Dopamine Adsorption and Charge Transfer Kinetics at Glassy Carbon Surfaces
}

\author{
James A. Behan, ${ }^{a}$ Filip Grajkowski, ${ }^{a}$ Dilushan R. Jayasundara,${ }^{b}$ Laia Vilella-Arribas, ${ }^{a}$ \\ Max García-Melchor $*^{a}$ and Paula E. Colavita $*^{a}$ \\ ${ }^{a}$ School of Chemistry, CRANN and AMBER Research Centres, Trinity College Dublin, \\ College Green, Dublin 2, Ireland. \\ ${ }^{b}$ Department of Physics, University of Colombo, Colombo 03, Sri Lanka \\ "E-mail: garciamm@tcd.ie (M.G.-M.). E-mail colavitp@tcd.ie (P.E.C.).
}




\begin{abstract}
Abnormal levels of the neurotransmitter dopamine have been linked to a variety of neurochemical disorders including depression and Parkinson's disease. Dopamine concentrations are often quantified electrochemically using biosensors prepared from carbon electrode materials such as carbon paste or glassy carbon. The charge transfer kinetics of dopamine are highly sensitive to carbon surface termination, including the presence of certain oxygen functional groups and adsorption sites. However, the nature of the binding sites and the effects of surface oxidation on the voltammetry of dopamine are both poorly understood. In this work the electrochemical response of dopamine at glassy carbon model surfaces was investigated to understand the effects of altering both the carbon nanostructure and oxygen surface chemistry on dopamine charge transfer kinetics and adsorption. Glassy carbon electrodes with low oxygen content and a high degree of surface graphitisation were prepared via thermal annealing at $900{ }^{\circ} \mathrm{C}$, whilst highly oxidised glassy carbon electrodes were obtained through electrochemical anodisation at $1.8 \mathrm{~V}$ vs $\mathrm{Ag} / \mathrm{AgCl}$. The carbon surface structure and composition in each case was studied via X-Ray Photoelectron Spectroscopy. Voltammetry in solutions of dopamine at acidic $\mathrm{pH}$ confirmed that both annealing and anodisation treatments result in carbon surfaces with rapid charge transfer kinetics. However, dopamine adsorption occurs only at the low-oxygen, highly-graphitized carbon surface. Density functional theory studies on graphene model surfaces reveal that this behaviour is due to non-covalent interactions between the $\pi$-system of dopamine and the basal sites in the annealed surface. Simulations also show that the introduction of oxygen moieties disrupt these interactions and inhibit dopamine adsorption, in agreement with experiments. The results clarify the role of oxygen moieties and basal plane sites in facilitating both the adsorption of and charge transfer to DA at carbon electrodes.
\end{abstract}




\section{Introduction}

Catechols are an important class of biomolecules with particular relevance to neurochemistry. Abnormal levels of catecholamines such as dopamine (DA) have been linked to psychiatric disorders such as depression [1, 2], schizophrenia [3], and Parkinson's disease [4]. The elevation of DA levels in the synapses of dopaminergic neurons by stimulant use has also been implicated in the pathogenesis of addiction [5].

The quantification of catechol concentrations both in vivo and through the ex situ analysis of biological fluids has often been accomplished electrochemically as reviewed recently by Jacowska and Krysinski [6]. Carbon materials have been applied as biosensors for catecholamines because of their low cost, biocompatibility, and versatility. Amperometric sensors for DA based on carbon paste electrodes [7], carbon fibers [8], boron doped diamond [9], and glassy carbon [10] have been reported. Due to the interference of co-analytes such as ascorbic acid, which has a similar reduction potential to catechols, researchers have employed methodologies such as the immobilisation of enzymes at the electrode surface, [9] doping the carbon scaffold with nitrogen $[11,12]$ and other pre-treatments of the carbon surface in order to improve selectivity.

Catechol oxidation is complex and has been described according to the 9-membered scheme of squares [13], which involves the net transfer of 2 protons and 2 electrons from the catechol $[14,15]$ as shown in Scheme 1 . The mechanism is pH-dependent; for instance, under basic conditions the pathway involves the transfer of 2 electrons from a deprotonated catechol $\mathrm{C}^{2-}$ to form a quinone species $\mathrm{C}$ as shown in the top row of the scheme. At low $\mathrm{pH}$ the catechol is expected to exist predominantly in its monoprotonated $\mathrm{CH}^{-}$form or the diprotonated $\mathrm{CH}_{2}$ form, implying that proton transfers must occur at some point in the reaction mechanism. The order of the reaction steps at $\mathrm{pH}=1$ is known to be e- $\mathrm{H}^{+}-\mathrm{e}-\mathrm{H}^{+}[16]$. 


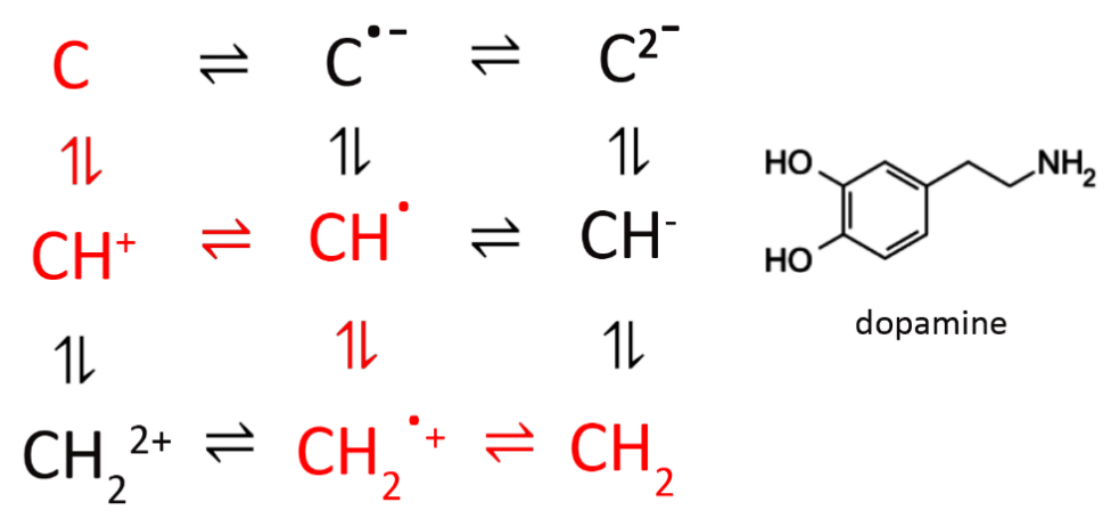

Scheme 1. 'Scheme of squares' illustrating possible pathways for the 2-electron, 2-proton oxidation of a generic catechol $\left(\mathrm{CH}_{2}\right)$. The pathway taken under low $\mathrm{pH}$ conditions is highlighted in red [17]. The chemical structure of dopamine is shown on the right hand side of the scheme.

In addition to the complex reaction scheme, the kinetics of catechol oxidation at carbon surfaces are known to be highly sensitive to the electrode preparation, including the polishing and cleaning steps, exposure to organic solvents and any surface pre-treatments employed. For glassy carbon (GC) the literature is replete with methodologies for surface pretreatments, both electrochemical and otherwise, which produce surfaces with properties amenable to fast charge-transfer kinetics. However, it is difficult to identify which of these protocols (if any) is the most effective, as reports in the literature are often contradictory; for instance, electrochemical oxidation of GC has been reported to induce catechol adsorption by some groups [18]whilst others have reported a positive effect on the electron transfer kinetics after oxidative treatments in the absence of adsorption [19].

It is generally thought that adsorption facilitates the inner-sphere redox process via a self-catalytic process which was proposed to involve interactions between adsorbates and solution-phase catechol. Previously [20] we have shown that dopamine adsorption at graphitised carbon surfaces occurs most favourably at basal regions of the carbon scaffold 
through non-covalent $\pi$-stacking interactions, and that this adsorption is correlated to rapid charge transfer kinetics to solution phase DA. Although the precise self-catalytic mechanism is uncertain, hydrogen-bonding interactions involving the hydroxyl groups of catechol and the $\mathrm{C}=\mathrm{O}$ of adsorbed quinone have been suggested [18]. The chemisorption of blocking organic monolayers which impede catechol adsorption has an inhibitory effect on the kinetics of charge transfer to catechols, an important exception being when the chemical structure of the adsorbed species resembles a quinone [21]. In the case of a quinone-like layer, adsorption of the catechol from solution is inhibited but the electron transfer to dopamine in solution is quasi-reversible. This suggests that it might be possible to achieve a similar enhancement of charge transfer rates via GC pre-treatments, so long as these result in interfacial interactions similar to those obtained via a quinone adlayer. In particular oxidative treatments might offer a successful route towards activation if such protocols also introduce quinone-mimicking moieties at the electrode surface.

In this work we carry out a detailed investigation of the effects of oxidative pretreatments on the activation of GC electrodes towards dopamine adsorption and electrooxidation. Effects of surface oxidation on catechol electrochemistry have been observed in the literature, however it remains unclear which chemical and structural properties of the oxidised surface result in the changes observed, thus resulting in relatively unpredictable catechol responses. Our study is carried out by preparing GC electrodes with well-defined surface chemistry via pre-treatment protocols and correlating the properties of each surface to the charge transfer kinetics. The chosen pre-treatment protocols result in surfaces with different surface properties and radically different oxygen contents [22].

The electron transfer kinetics of DA on each GC surface are evaluated using cyclic voltammetry and these results are correlated to the surface composition as determined from 
X-ray Photoelectron Spectroscopy (XPS) measurements. Results indicate that DA adsorption does not occur on highly oxidised GC surfaces prepared via anodisation, although charge transfer occurs quasi-reversibly at these surfaces. Thermal annealing produces a low-oxygen content surface on which DA readily adsorbs, resulting in reversible charge transfer kinetics to the catechol in solution. Based on a careful analysis of the $\mathrm{C} 1 \mathrm{~s}$ envelopes of each surface, the tendency for both catechols to adsorb only on the annealed surface is strongly correlated to the graphitisation of the GC surface induced by the heat treatment.

Conclusions derived from the experimental results are supported by DFT calculations on model carbon clusters based on pristine graphene and graphene terminated with different oxygen moieties at varying coverage to obtain molecular level detail on binding interactions in the presence of specific moieties. Importantly, the combined experimental and computational findings clarify the role of oxidation and basal plane sites in facilitating DA redox processes at carbon electrodes. Fast charge transfer can be achieved via both adsorbate-catalysed and H-bonding-catalysed mechanisms, thus highlighting the ability to tailor the interfacial charge transfer mechanisms via anodisation/graphitisation of the carbon electrode.

\section{Experimental Methods}

\section{Chemicals and Materials}

Dopamine hydrochloride (98\%, Aldrich), Sulfuric Acid ( $\geq 95 \%$, Ultratrace, Aldrich), Potassium Hydroxide (Ultratrace, Aldrich) and Methanol (Semiconductor Grade) were used without further purification.

\section{Substrate Preparation}

Glassy carbon (GC) discs (HTW Sigradur radius $0.25 \pm 0.05 \mathrm{~cm}$ ) were prepared by polishing with progressively finer grades of alumina slurry (Buehler) with particle sizes of 1 
$\mu \mathrm{m}, 0.3 \mu \mathrm{m}$ and $0.05 \mu \mathrm{m}$ on microcloth (Buehler). Between each round of polishing the disks were rinsed with copious millipore water, then sonicated in methanol and millipore water for 3 minutes each. After the final polishing step this sonication was increased to 10 minutes in each solvent, followed by drying under Ar. The polished discs were either used immediately for electrochemical measurements or subjected to electrochemical/thermal pre-treatments. Annealed GC surfaces were prepared by placing the polished GC discs in a tube furnace at $900{ }^{\circ} \mathrm{C}$ under $\mathrm{N}_{2}$ for $1 \mathrm{~h}$. Anodised GC was prepared by mounting the disc in a static Teflon electrode holder and applying a potential of $1.8 \mathrm{~V}$ vs $\mathrm{Ag} / \mathrm{AgCl}$ (sat) in an aqueous solution of $0.1 \mathrm{M} \mathrm{KOH}$ for $20 \mathrm{~s}$.

\section{Electrochemical Characterisation}

Electrochemical measurements were carried out using a Metrohm Autolab AUT50324 potentiostat using a 3-electrode setup. A static teflon disc holder (Pine Instruments) enclosing the GC discs was used as working electrode. A Hydroflex hydrogen electrode (Gaskatel) and graphite rod were used as reference and counter electrodes respectively. The electrochemical cell was a five-necked jacketed cell (Pine Instruments) which had its temperature held constant at $25{ }^{\circ} \mathrm{C}$ using a recirculator. Prior to experiments the cell was cleaned using Piranha solution (3:1 $\mathrm{H}_{2} \mathrm{SO}_{4}: \mathrm{H}_{2} \mathrm{O}_{2}$ CAUTION: Piranha solution is a strong oxidant which may react explosively with organic solvents and must always be used in a fumehood) followed by rinsing with copious amounts of Millipore water. The cell was then rinsed three times with the electrolyte solution used during the experiment immediately prior to the analysis.

Cyclic Voltammograms (CVs) were acquired in a potential window of $0.48-1.2 \mathrm{~V}$ vs RHE in deaerated solutions of $0.1 \mathrm{M} \mathrm{H}_{2} \mathrm{SO}_{4}$ with and without DA in concentrations ranging from $25 \mu \mathrm{M}$ to $1 \mathrm{mM}$. All voltammograms were taken with $\mathrm{iR}$ compensation using 
commercial software (NOVA) with the uncompensated resistance of $18 \pm 1 \Omega$ determined prior to the experiment using Electrochemical Impedance Spectroscopy (EIS).

\section{X-Ray Photoelectron Spectroscopy Analysis of GC Surfaces}

$\mathrm{X}$-ray photoelectron spectroscopy (XPS) characterisation was performed at $1 \times 10^{-10}$ mbar base pressure in an ultrahigh-vacuum system (Omicron). The X-ray source was a monochromatised $\mathrm{Al} \mathrm{K \alpha}$ source $(1486.6 \mathrm{eV})$. Spectra were recorded at $45^{\circ}$ takeoff angle with an analyser resolution of $0.5 \mathrm{eV}$. Spectra were baseline corrected using a Shirley background and fitted with Voigt functions using commercial software (CasaXPS 2.3.17); atomic percent compositions were determined by calculating peak area ratios from high resolution spectra after correction by relative sensitivity factors (RSF, C $1 \mathrm{~s}=1.0, \mathrm{~N} 1 \mathrm{~s}=1.8, \mathrm{O} 1 \mathrm{~s}=2.93$ ).

\section{Computational Studies}

Density functional theory (DFT) calculations were carried out using the dispersion corrected hybrid functional $\omega$ B97X-D developed by Head-Gordon and Chai [23], implemented in the Gaussian09 software package [24]. This level of theory has provided satisfactory results in previous theoretical studies of non-covalent interactions $[20,25] . \mathrm{H}$ atoms were described using the double- $\zeta$ basis set $6-31 \mathrm{G}(\mathrm{d}, \mathrm{p})$, whereas the same basis set plus diffuse functions was employed to describe the more electronegative $\mathrm{O}$ and $\mathrm{N}$ atoms. No constraints were imposed during geometry optimisations and the nature of all resulting stationary points was further verified through vibrational frequency analysis. Adsorption Gibbs energies $\left(\Delta \mathrm{G}_{\mathrm{ads}}\right)$ for DA on model graphene clusters were calculated at the experimental temperature of $298 \mathrm{~K}$ and pressure of $1 \mathrm{~atm}$, according to the following equation:

$$
\Delta \mathrm{G}_{\mathrm{ads}}=\mathrm{G}_{\text {surf }+\mathrm{DA}}-\mathrm{G}_{\text {surf }}-\mathrm{G}_{\mathrm{DA}}
$$


where $G_{\text {surf+DA }}, G_{\text {surf }}$, and $G_{D A}$ correspond to the adsorbed DA on the graphene cluster, the clean graphene cluster, and the DA molecule in the gas phase, respectively. Intermolecular forces between the adsorbed DA and the graphene model surfaces were investigated via non-covalent interaction (NCI) analysis based on the behaviour of the reduced density gradient at low electron densities using the NCIPLOT program [26, 27].

\section{Results and Discussion}

In order to investigate the effects of surface treatments on catechol kinetics, glassy carbon electrodes were prepared using three different methodologies. GC discs used for electrochemical studies immediately after polishing are referred to as 'polished GC.' Two other surface treatments were also employed to induce significant structural and chemical changes in the GC surface after the polishing step. The first treatment was an oxidation via electrochemical anodisation at $1.8 \mathrm{~V}$ vs $\mathrm{Ag} / \mathrm{AgCl}$ (sat) in $0.1 \mathrm{M} \mathrm{KOH}$ for $20 \mathrm{~s}$ ('anodised GC'). The second treatment involved thermal annealing under inert atmosphere in a tube furnace at $900{ }^{\circ} \mathrm{C}$ for $1 \mathrm{~h}$ ('annealed GC'). The chemical composition of all three of these surfaces was studied using XPS, whilst the charge transfer kinetics in DA solutions were evaluated using cyclic voltammetry experiments.

\subsection{Cyclic Voltammetry of Dopamine on GC Surfaces}

A typical voltammogram obtained at polished $\mathrm{GC}$ in $1 \mathrm{mM} \mathrm{DA} / 0.1 \mathrm{M} \mathrm{H}_{2} \mathrm{SO}_{4}$ is shown in Figure 1a. A broad waveform with a large peak-to-peak separation $(\Delta \mathrm{E}=220 \mathrm{mV})$ is obtained even at the low scan rate of $5 \mathrm{mV} \mathrm{s}^{-1}$, suggesting that charge transfer to DA is electrochemically irreversible after our polishing protocol. The peaks arise from DA alone as confirmed by a control CV in supporting electrolyte (dashed line). 

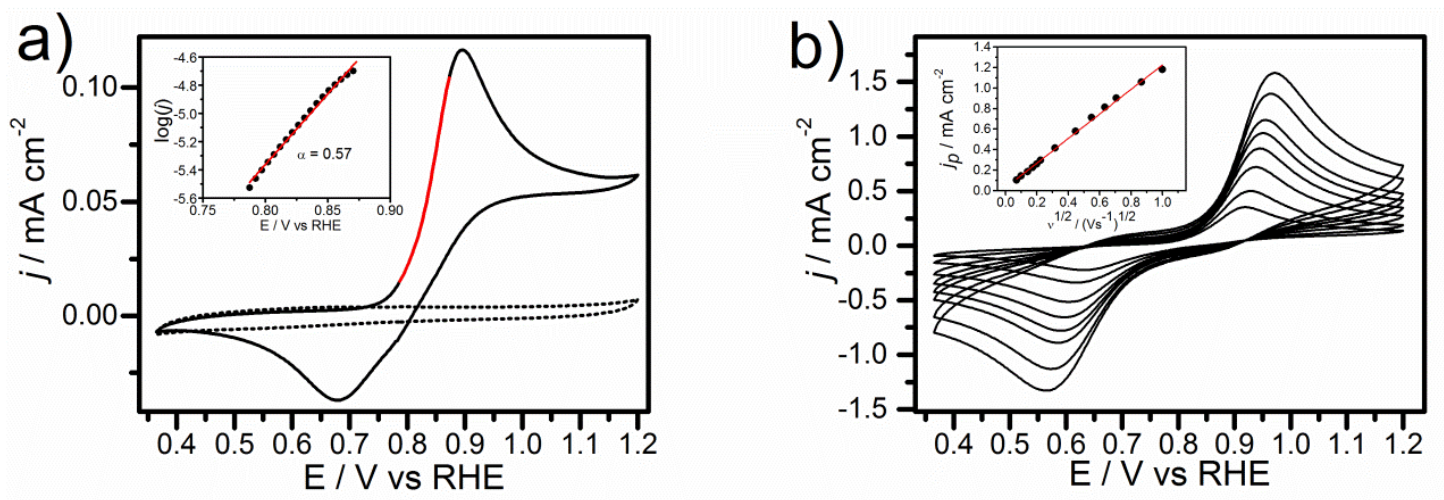

Figure 1. (a) Cyclic voltammogram of polished $\mathrm{GC}$ in $1.0 \mathrm{mM}$ DA in $0.1 \mathrm{M} \mathrm{H}_{2} \mathrm{SO}_{4}$ at $5 \mathrm{mV}$ $\mathrm{s}^{-1}$. The dashed line shows the voltammogram in $0.1 \mathrm{M} \mathrm{H}_{2} \mathrm{SO}_{4}$ supporting electrolyte. The inset shows a Tafel plot of the region highlighted in red on the voltammogram. (b) Voltammograms of GC in $1.0 \mathrm{mM}$ DA / $0.1 \mathrm{M} \mathrm{H}_{2} \mathrm{SO}_{4}$ at scan rates between $5 \mathrm{mV} \mathrm{s}^{-1}$ and 1 $\mathrm{V} \mathrm{s}^{-1}$. The inset shows a plot of peak current density, $j_{\mathrm{p}}$, on the anodic scan versus the square root of the scan rate.

The broad, asymmetric waveform for DA oxidation and reduction in Figure 1a on polished GC and similar carbon electrode materials has been observed previously [16, 28]. Referring to the scheme-of-squares for DA oxidation, it is consistent with a mechanism where the first electron transfer is the rate determining step [29]. This conclusion is supported by Tafel analysis of the rising part of the wave on the anodic sweep as highlighted in Figure 1a and plotted in the inset. The Tafel slope of the linear fit to this data results in a value of the apparent transfer coefficient, $(\mathrm{n}+\alpha)=0.57$, which is close to the value of 0.5 expected for an overall $2 \mathrm{e}^{-}$process where the first electron transfer step is rate determining $[15,17,30]$.

$\mathrm{CVs}$ of polished GC in $1 \mathrm{mM}$ DA solution at various scan rates are shown in Figure $1 \mathrm{~b}$ with the relevant redox parameters summarised in Table 1 . The value of $\Delta \mathrm{E}$ increases with increasing scan rate as expected for an irreversible charge transfer process. The peak current density, $j_{p}$, increases with the square root of the scan rate, $v^{1 / 2}$ as shown in the inset of the figure. Using the value of $0.196 \mathrm{~cm}^{2}$ for the electrode area, $\alpha=0.5$ and $6.6 \times 10^{-6} \mathrm{~cm}^{2} \mathrm{~s}^{-1}$ for the diffusion coefficient of DA determined from rotating disk electrode measurements, (See 
Supporting Information) the resulting slope of $0.0012 \mathrm{~mA} \mathrm{~cm}^{-2}\left(\mathrm{~V} \mathrm{~s}^{-1}\right)^{-1 / 2}$ is consistent with an irreversible 2 electron charge transfer process according to equation (1) [27]:

$$
j_{p}=0.496 \sqrt{n+\alpha} n F c \sqrt{\frac{F v D}{R T}}
$$

The oxidation of GC has been previously observed to result in improvements in DA electron-transfer kinetics [29, 31]Furthermore, anodic treatments, as reported by McDermott et al. [32], are known to have a "cleaning" effect on polished GC surfaces, whereby oxidation results in removal of polishing debris, which can block active sites for charge transfer processes. A similar cleaning effect has also been observed on a variety of carbon electrode materials used for the electrochemical detection of dopamine [33-35]. The response of an anodised GC electrode was therefore tested in $1 \mathrm{mM} \mathrm{DA} / 0.1 \mathrm{M} \mathrm{H}_{2} \mathrm{SO}_{4}$ at a scan rate of 5 $\mathrm{mV} \mathrm{s}^{-1}$, as shown in Figure 2a. The voltammogram has a small $\Delta \mathrm{E}$ value of $c a .40 \mathrm{mV}$ (Table 1) which is close to the theoretical value of $59 / 2 \mathrm{mV}$ for a reversible 2e charge transfer, suggesting that the anodisation protocol results in a GC surface with quasi-reversible kinetics. The peak current densities of the cathodic $\left(j_{p, c}\right)$ and anodic $\left(j_{p, a}\right)$ scans are similar, yielding $j_{p},{ }_{d} j_{p, a}=0.86$, i.e. close to the ideal value of 1 for a fully reversible process. A Tafel plot of the rising part of the anodic peak (inset) yields $(\mathrm{n}+\alpha) \approx 1$, as expected for a reversible $2 \mathrm{e}^{-}$process [29]. Figure $2 \mathrm{~b}$ shows CVs obtained at anodised GC in $1 \mathrm{mM}$ DA / $0.1 \mathrm{M} \mathrm{H}_{2} \mathrm{SO}_{4}$ at scan rates up to $0.5 \mathrm{~V} \mathrm{~s}^{-1}$. The $\Delta \mathrm{E}$ increases slightly with scan rate but remains generally close to $40 \mathrm{mV}$. The plot of peak current density versus $v^{1 / 2}$ (inset) yields a slope of $2.3 \times 10^{-3}$ $\mathrm{mA} \mathrm{cm}{ }^{-2}\left(\mathrm{~V} \mathrm{~s}^{-1}\right)^{-1 / 2}$ which is close to the value of $1.9 \times 10^{-3}$ obtained with equation (2) [36] for a reversible $2 \mathrm{e}^{-}$process at $298 \mathrm{~K}$ [37].

$$
j_{p}=0.446 n F c \sqrt{\frac{n F v D}{R T}}
$$



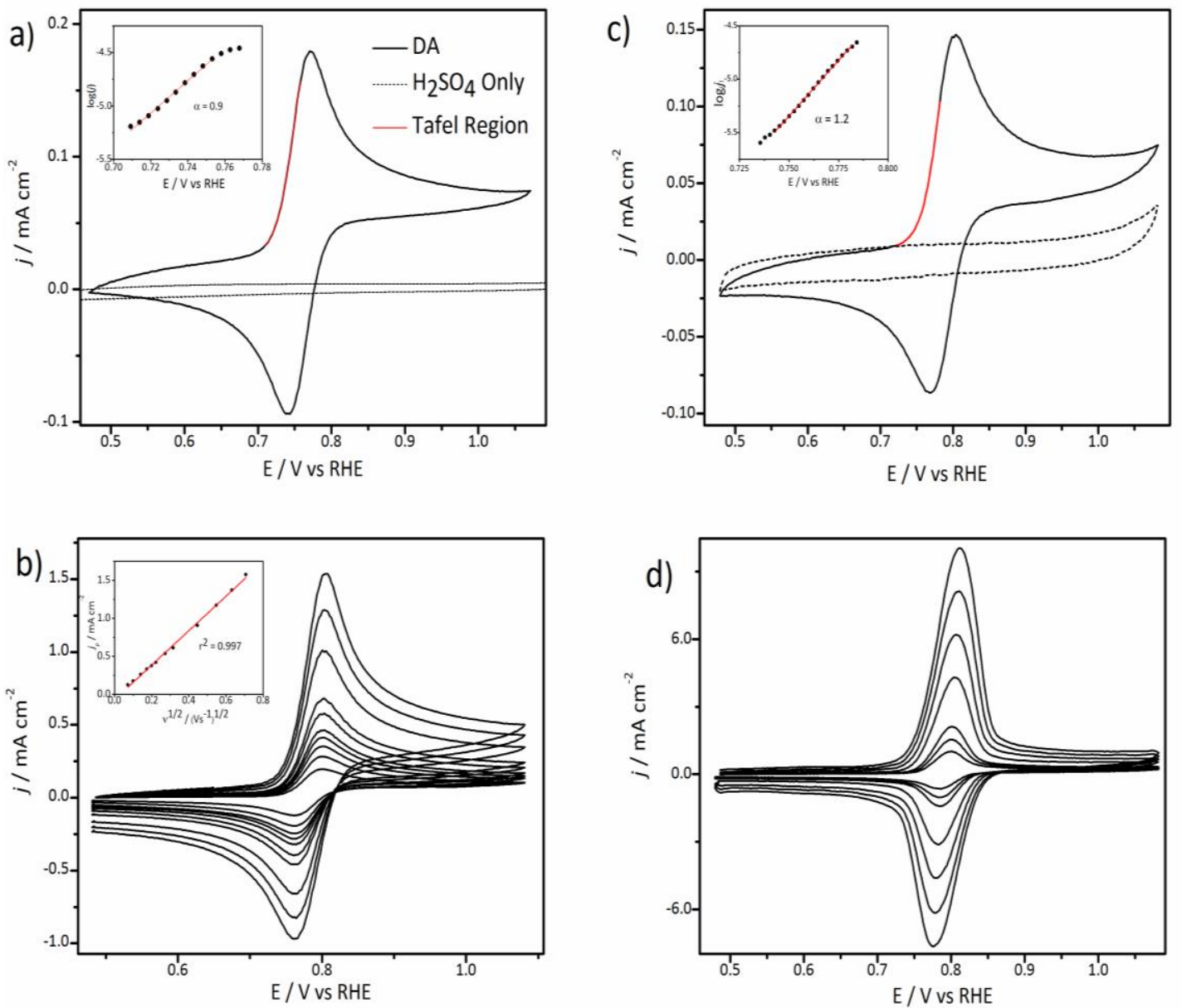

Figure 2. (a) Cyclic voltammogram of anodised $\mathrm{GC}$ in $1.0 \mathrm{mM}$ DA in $0.10 \mathrm{M} \mathrm{H}_{2} \mathrm{SO}_{4}$ and in $0.10 \mathrm{M} \mathrm{H}_{2} \mathrm{SO}_{4}$ supporting electrolyte at $5 \mathrm{mV} \mathrm{s}^{-1}$ (dotted line). The inset shows a Tafel plot derived from the highlighted region of the voltammogram. (b) Voltammograms of anodised $\mathrm{GC}$ at scan rates between 5 and $500 \mathrm{mV} \mathrm{s}^{-1}$ in $1.0 \mathrm{mM}$ DA in $0.1 \mathrm{M} \mathrm{H}_{2} \mathrm{SO}_{4}$. The inset shows peak current density versus $v^{1 / 2}$ derived from the voltammograms. (c) Voltammograms of annealed GC in $1.0 \mathrm{mM}$ DA in $0.1 \mathrm{M} \mathrm{H}_{2} \mathrm{SO}_{4}$ and in $0.1 \mathrm{M} \mathrm{H}_{2} \mathrm{SO}_{4}$ supporting electrolyte at 5 $\mathrm{mV} \mathrm{s}^{-1}$. The inset shows a Tafel plot derived from the highlighted region of the voltammogram. (d) Voltammograms of annealed GC in $1.0 \mathrm{mM} \mathrm{DA}$ in $0.1 \mathrm{M} \mathrm{H}_{2} \mathrm{SO}_{4}$ at scan rates between 50 and $500 \mathrm{mV} \mathrm{s}^{-1}$.

The anodisation pre-treatment was therefore found to be highly effective at producing a GC surface with rapid charge transfer kinetics. This conclusion is also supported by the estimate of the apparent charge transfer rate constant $\left(\mathrm{k}_{\mathrm{app}}^{0}\right)$ of $3 \times 10^{-3} \mathrm{~cm} \mathrm{~s}^{-1}$ as discussed in the Supporting Information. 
The third surface pre-treatment protocol used in this study involved thermal annealing of a polished GC disk, which is known to reproducibly yield clean GC surfaces [38]. Figure 2c shows a CV of annealed GC in $1 \mathrm{mM} \mathrm{DA} / 0.1 \mathrm{M} \mathrm{H}_{2} \mathrm{SO}_{4}$ at $5 \mathrm{mV} \mathrm{s}^{-1}$. The $\Delta \mathrm{E}$ value of $30 \mathrm{mV} \mathrm{s}^{-1}$ and a $j_{p}, c_{j} / j_{p, a}=0.91$ are both consistent with a reversible $2 \mathrm{e}$ process (Table 1 ). The Tafel plot (inset) further confirms this conclusion $(\alpha=1.2)$ as does the estimate of $0.05 \mathrm{~cm} \mathrm{~s}^{-1}$ for $\mathrm{k}_{\mathrm{app}}^{0}$ (Supporting Information). Therefore, thermal annealing was also found to be an effective surface preparation method for producing a carbon surface with rapid charge transfer kinetics to DA.
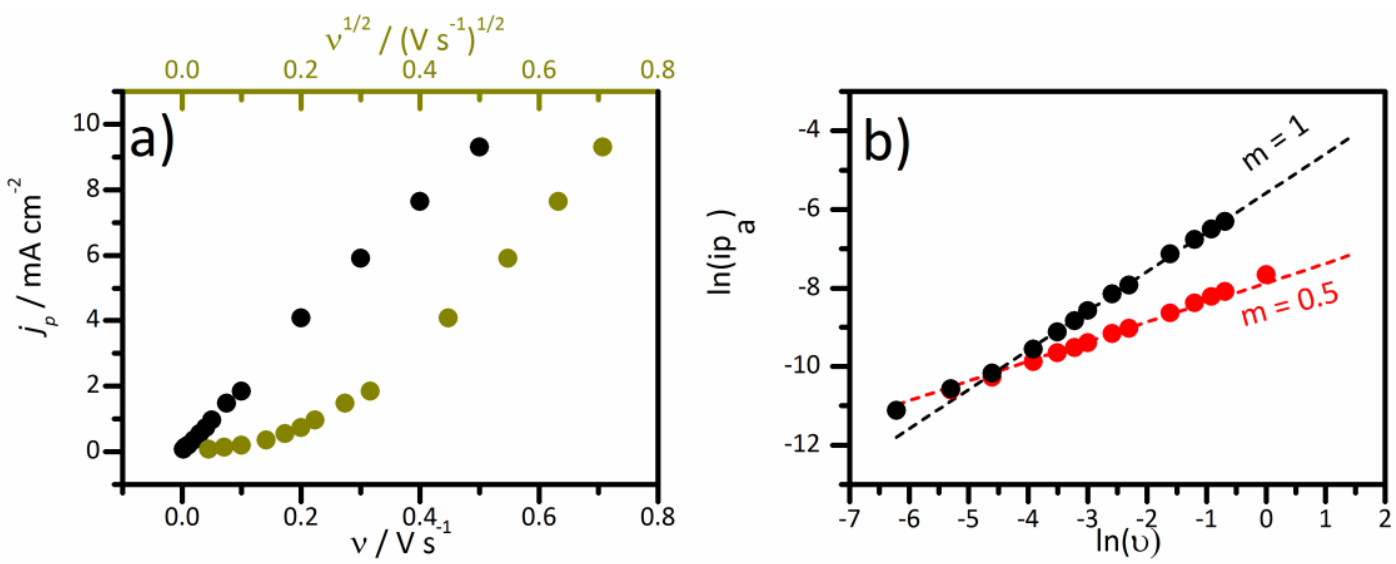

Figure 3. (a) Plots of peak current densities derived from the voltammograms in Figure 2 versus $v$ (black plot) and $v^{1 / 2}$ (gold plot). (b) Plot of $\ln \left(\mathrm{i}_{\mathrm{p}, \mathrm{a}}\right)$ versus $\ln (v)$ for annealed GC (black dots) and anodised GC (red dots). The dashed lines represent theoretical lines with slope $m=1$ and $m$ $=0.5$ respectively and are reported to guide the eye.

Table 1. Summary of voltammetry data for the oxidation of $1 \mathrm{mM} \mathrm{DA}$ in $0.1 \mathrm{M} \mathrm{H}_{2} \mathrm{SO}_{4}$ on GC electrodes with different surface preparation methods. $\Delta \mathrm{E}$ and peak current density data are taken from voltammograms carried out at a scan rate of $5 \mathrm{mVs}^{-1}(n=4)$.

\begin{tabular}{llll}
\hline Surface & $\mathbf{\Delta E} / \mathbf{m V}$ & $\mathbf{j}_{\mathbf{p}, \mathbf{c}} / \mathbf{j}_{\mathbf{p}, \mathbf{a}}$ & $\boldsymbol{\alpha}_{\text {eff }}$ \\
\hline Polished GC & $245 \pm 70$ & $0.5 \pm 0.4$ & $0.5 \pm 0.1$ \\
Annealed GC & $30 \pm 3$ & $0.89 \pm 0.03$ & $1.0 \pm 0.3$ \\
Anodised GC & $40 \pm 3$ & $0.85 \pm 0.04$ & $0.8 \pm 0.1$ \\
\hline
\end{tabular}


As discussed in the introduction, rapid electron transfer to catechols has been attributed to a 'self-catalysis' mechanism [21] whereby adsorption of catechol at the electrode surface facilitates electron transfer to catechols in the solution phase. Adsorbed catechols are themselves redox active $[16,39]$ and their contribution to the Faradaic peaks can be enhanced by increasing the scan rate [40]. Figure $2 \mathrm{~d}$ shows $\mathrm{CV}$ s of annealed GC in $1 \mathrm{mM}$ DA / $0.1 \mathrm{M} \mathrm{H}_{2} \mathrm{SO}_{4}$ at varying scan rates. Voltammograms obtained up to rates close to $50 \mathrm{mV} \mathrm{s}^{-1}$ have a similar form to the $\mathrm{CV}$ obtained at $5 \mathrm{mV} \mathrm{s}^{-1}$ (Figure 2c) i.e. they have $\Delta \mathrm{E}$ values close to $30 \mathrm{mV}$. However, for larger scan rates the voltammogram gradually becomes more symmetric in shape around the formal potential, $\mathrm{E}^{0^{\prime}}=0.78 \mathrm{~V}$ vs RHE, and $\Delta \mathrm{E}$ reduces in magnitude down to values $<15 \mathrm{mV}$. This behaviour is consistent with the presence of surface-bound DA.

The peak current densities were found to be linear vs the scan rate, $v$, rather than $v^{1 / 2}$ (Figure 3a) as expected for an adsorbed redox species according to equation 3 [41]:

$$
i_{p}=\frac{n^{2} F^{2} v A \Gamma}{4 R T}
$$

where $\Gamma$ is the surface coverage of DA in $m o \mathrm{~cm}^{-2}$ and $A$ is the electrode area in $\mathrm{cm}^{2}$. Using Equation 3, the estimated value of $\Gamma$ for DA at Annealed GC is $5 \times 10^{-9} \mathrm{~mol} \mathrm{~cm}{ }^{-2}$, a value which is largely consistent with previous studies of DA adsorption at carbon electrode surfaces [20,42]. The Faradaic current at the annealed GC surface is therefore comprised of contributions from both adsorbed DA and solution-phase DA, with the relative contributions of each distinguished from one another by the scan rate employed during the experiment [20].

The contributions arising from adsorbed DA species can be compared using a log-log plot of the peak current $\left(i_{p}\right)$ vs scan rate for the two electrodes that yield quasi-reversible behaviour, i.e. anodised and annealed GC, as shown in Figure 3b. For anodised GC, the scatter plot falls on a theoretical line of slope $m=0.5$, consistent with a diffusion-controlled 
process. For annealed GC the plot displays $m=0.5$ at low scan rates but deviates significantly as the scan rate increases, displaying instead $m=1$ at $>100 \mathrm{mV} \mathrm{s}^{-1}$, which indicates that in this regime $i_{p}$ is dominated by contributions from adsorbed DA. The crossover point from diffusion to adsorption predominance depends on the concentration of DA in solution as well as the scan rate. For instance, in $25 \mu \mathrm{M}$ DA the measured peak currents are proportional to $v$ across the entire scan rate range (Supporting Information).

The response of the three different types of GC surfaces in solutions of DA is summarised in Table 1. Both anodisation and annealing pretreatments are effective in producing surfaces with rapid charge transfer kinetics to DA; however, the mechanism of activation does not appear to be identical on both surfaces. The anodised surface exhibits facile charge transfer to DA with no evidence for the presence of DA adsorbates. However, the annealed surface clearly favours the adsorption of DA, which may explain the rapid charge transfer kinetics via a self-catalytic mechanism.

\subsection{X-Ray Photoelectron Spectroscopy Analysis of GC Surfaces}

To understand the observed differences between anodised and annealed GC their surfaces were characterised via XPS. Survey scans of anodised and annealed GC electrodes, together with that of polished GC, are reported in the Supporting Information. C 1s and O 1s contributions are the only ones present in all three surfaces. There are however obvious differences in the oxygen content among the three spectra, as shown by the $\mathrm{O} / \mathrm{C}$ at $\%$ derived from the $\mathrm{O}$ 1s / C 1s area ratios after normalisation by RSF (Table 2). Both polished GC and annealed GC have similar $\mathrm{O} / \mathrm{C}$ at $\%$ of $3 \pm 2 \%$, whereas the anodised disk has a much higher $\mathrm{O} / \mathrm{C} \%$ of $13 \pm 3 \%$. The small oxygen contributions on annealed and polished GC are likely attributable to adventitious oxygen contamination from air exposure [43], however the large 

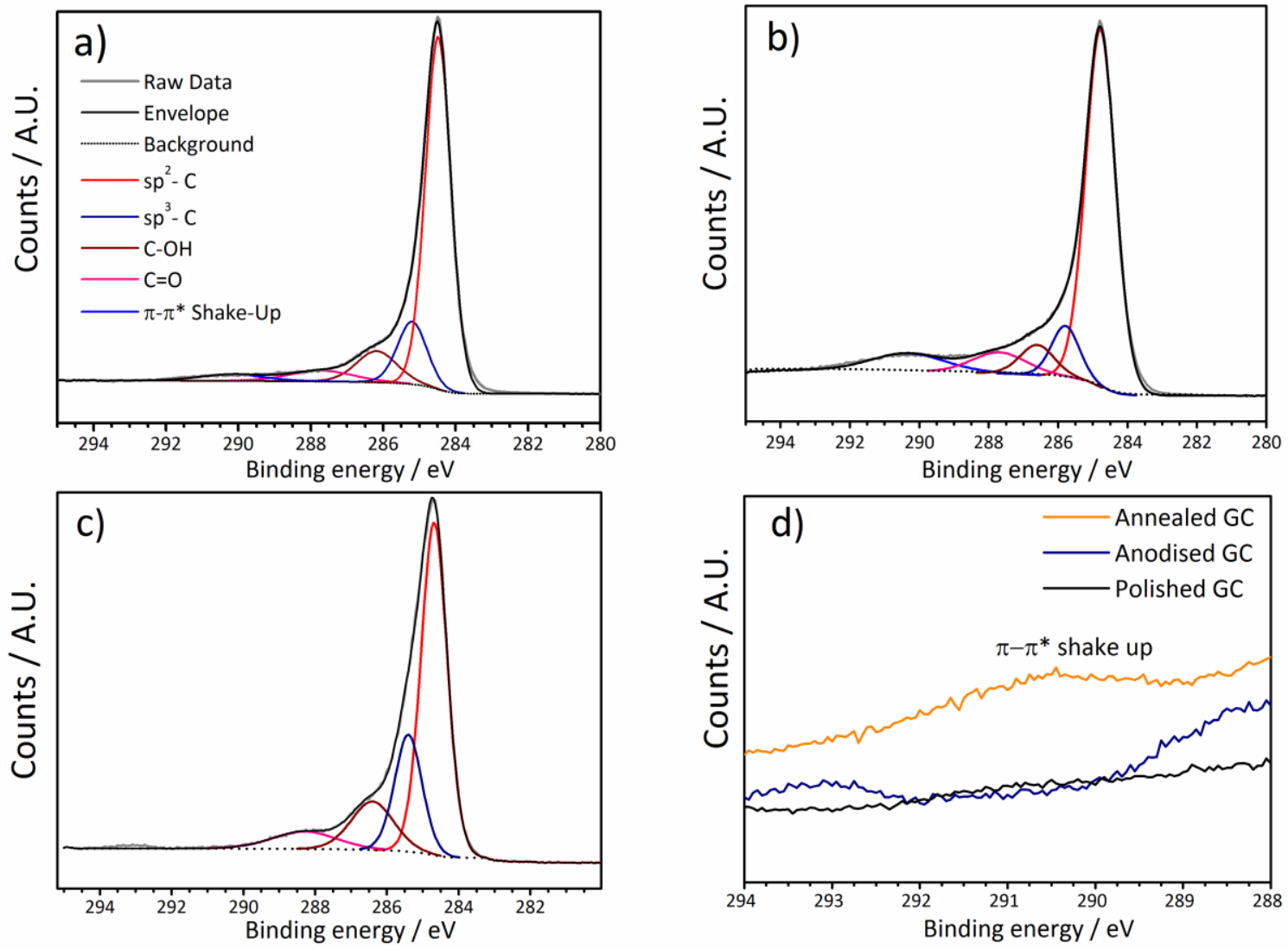

Figure 4. C 1s spectra of (a) polished GC (b) annealed GC (c) anodised GC, and their best-fit deconvolution. The raw data in each case is shown by a continuous gray line, while the envelope resulting from the deconvolution is overlaid in black in each case. (d) shows expanded C 1s spectra in the 294-288 eV region; annealed, anodised and polished GC are shown as orange, navy and black traces respectively.

value observed for anodised GC must arise from chemical changes resulting from the oxidative pre-treatment $[44,45]$.

The $\mathrm{C}$ 1s envelopes are plotted in Figure 4. All three surfaces show an asymmetric peak at ca. $284.6 \mathrm{eV}$ which is typical for a graphitic carbon surface. Polished GC (Figure 4a) was deconvoluted into five main contributions assigned to $\mathrm{sp}^{2}$-centers $(284.6 \mathrm{eV}), \mathrm{sp}^{3}$-centers $(285.6 \mathrm{eV}), \mathrm{C}-\mathrm{OH}(286.5 \mathrm{eV})$ and $\mathrm{C}=\mathrm{O}(287.5 \mathrm{eV})[46,47]$. Finally, a broad peak at approximately $290 \mathrm{eV}$ is attributed to the $\pi-\pi^{*}$ shake-up feature of the delocalised graphitic network [47]. Annealed GC (Figure 4b) has a similar deconvolution to the polished surface, although the $\mathrm{sp}^{3}-\mathrm{C}$ and oxide peaks are lower in magnitude while the $\pi-\pi^{*}$ shake-up feature is 
significantly enhanced. This is consistent with increased graphitisation induced by thermal annealing. Anodised GC (Figure 4c) shows larger contributions from $\mathrm{sp}^{3}-\mathrm{C}$ at $285.6 \mathrm{eV}$ as well as from C-oxide peaks over the range $286-289 \mathrm{eV}$, as expected after oxidative treatments [44]. Notably, there is no evidence of a shake-up feature at $290 \mathrm{eV}$, which indicates that the delocalised graphitic network is severely disrupted by anodisation. This is more clearly shown in Figure 4d, which shows an expanded version of the shake-up region (295-288 eV). It is clear that the $\pi-\pi^{*}$ peak is absent in anodised GC while it is prominent in annealed GC. We note that the small contribution at $c a .293 \mathrm{eV}$ is typically attributed to intercalation of potassium [48] which is likely to arise from the anodisation in $\mathrm{KOH}$. Finally, the proportion

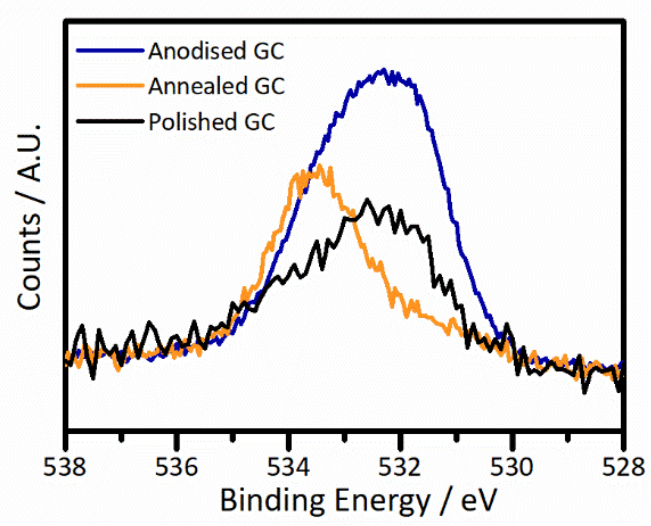

Figure 5. O 1s spectra for anodised, annealed and polished GC surfaces.

Table 2. Selected composition data derived from XPS measurements on GC surfaces. $(n=3)$

\begin{tabular}{cccc}
\hline Surface & O/C at\% & C-oxide $\%$ & $\mathrm{sp}^{3} / \mathrm{sp}^{2} \%$ \\
\hline Polished GC & $3 \pm 2$ & $14 \pm 3$ & $20 \pm 2$ \\
Annealed GC & $3 \pm 2$ & $19 \pm 4$ & $13 \pm 1$ \\
Anodised GC & $13 \pm 3$ & $28 \pm 4$ & $35 \pm 2$ \\
\hline
\end{tabular}


of contributions at $286.5-287.5 \mathrm{eV}$ arising from oxidised groups relative to the total area (Coxide, Table 2) closely follows the trends in $\mathrm{O} / \mathrm{C}$ at $\%$ calculated from $\mathrm{O} 1 \mathrm{~s}$ and $\mathrm{C} 1 \mathrm{~s}$ peaks. The $\mathrm{O}$ 1s spectra for the three GC surfaces are plotted in Figure 5 with deconvoluted spectra presented in the Supporting Information, Figure S4. Contributions to the envelope may be divided into two broad regions: $\mathrm{C}-\mathrm{O}$ in alcohols, ethers and esters between 533.5$535 \mathrm{eV}$ and $\mathrm{C}=\mathrm{O}$ in ketones, carboxylic acids and esters between 531.5-532.6 eV [36, 38, 40]. The peak shape for both anodised and polished GC is similar, suggesting that both of these surfaces possess a mix of oxide groups. The annealed surface, however, displays only a small contribution from $\mathrm{C}=\mathrm{O}$ groups.

Overall, the XPS data shows significant differences in surface structure and chemical composition among the three GC surfaces. Anodisation increases the content of surface oxides and $\mathrm{sp}^{3}$-centers, both of which are expected to result in interruptions of the delocalised graphitic network. This is further confirmed by the absence of a $\pi-\pi^{*}$ shake-up feature in the C 1s envelope for anodised GC. Thermal annealing results instead in increased surface graphitisation as shown by the low $\mathrm{sp}^{3} / \mathrm{sp}^{2}$ ratio and a prominent $\pi-\pi^{*}$ feature.

The voltammetry data discussed above show that annealed GC readily adsorbs DA, while anodised GC does not. Adsorption is known to promote fast kinetics of DA oxidation through self-catalysis at the electrode, as shown by McCreery et al. [21] Given that XPS results indicate that annealing leads to surface graphitisation producing a more extensive delocalised $\mathrm{sp}^{2}$ network than that of anodised GC, it appears likely that the binding sites for DA adsorption at the GC surface could be the graphitised cluster regions of the GC surface. These regions could interact with the catechol via $\pi$-interactions with the aryl moiety, as previously shown in the case of amorphous carbon electrodes [20], thus resulting in fast kinetics for DA at annealed GC. 
In our experiments, however, both anodised GC and annealed GC yield fast kinetics despite adsorption being detected at annealed GC exclusively, i.e. fast kinetics are decoupled from DA adsorption at anodised GC. McCreery et al. [21] had in fact observed that fast electron transfer can be achieved despite a lack of self-catalysis in the case of adlayers of quinones/catechols, and proposed that DA/catechol H-bonding interactions might be responsible for a reduction in the reorganisation energy. We therefore hypothesised that fast kinetics at anodised GC could be the result of favourable DA/electrode interactions with surface oxidised groups. To test this hypothesis we used computational methods to study DA interaction with graphitic clusters containing a range of oxidised groups, as discussed in the next section.

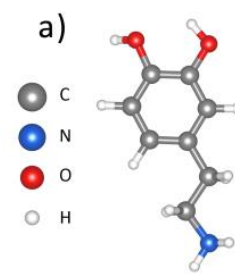

b)

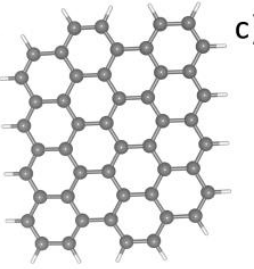

e)

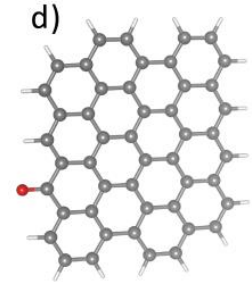

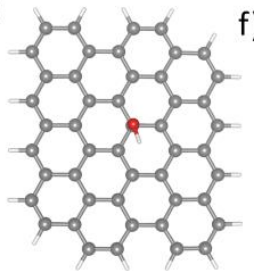

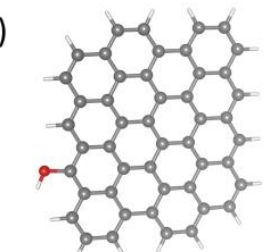

f)

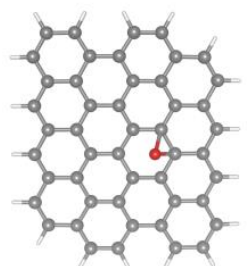

Figure 6. Structures used in DFT calculations for DA adsorption. (a) DA (b) Graphene cluster. (c) Graphene cluster with an edge hydroxyl moiety. (d) Graphene cluster with a carbonyl moiety. (e) Graphene cluster with a basal hydroxyl moiety. (f) Graphene cluster with an epoxide moiety.

\subsection{Computational Studies of DA Adsorption on Model Graphene Surfaces}

The interaction between DA (Figure 6a) and model surfaces based on graphitized carbon was studied at the DFT-wb97xd level, following a similar approach adopted in our previous work [20]. The model surfaces, shown in Figure 6b-f, consisted of graphene clusters with a 
fixed dimension and different defects introduced at either the edges, i.e. hydroxyl (6c) and carbonyl moieties (6d), or at the basal plane, i.e. hydroxyl (6e) and epoxide groups (6f). The computational analysis involved the optimisation of each carbon surface in isolation and then with DA adsorbed in a number of different orientations to calculate the lowest free energy of adsorption, $\Delta \mathrm{G}_{\text {ads }}$. These values along with the resulting orientation obtained are summarised in Figure 7. All of the orientations attempted along with the initial and final structures are given in the Supporting Information.

For the interaction of DA with the basal plane of a graphene fragment (DA-BP), the most favourable orientation of the molecule is parallel to the surface at a distance of $c a .3 .2 \AA$ (Figure 7a), as expected for a non-covalent $\pi$-interaction [20]. Subsequent examination of the interaction between DA and graphene clusters with oxidised moieties located at the edges (DA-CO and DA-OH-E, Figures $7 \mathrm{~b}-\mathrm{c}$ ) resulted in only minor changes in $\Delta \mathrm{G}_{\mathrm{ads}}$ relative to DA-BP. Notably, both optimized DA-CO and DA-OH-E structures preserved the interaction of the $\pi$-system of DA with the basal plane of the carbon surface, but in the case of DA-CO, the optimised structure also featured a relatively weak $\mathrm{H}$-bond interaction $(\mathrm{O}-\mathrm{H}$ bond length $=0.97 \AA$ ) between the carbonyl surface group and the $-\mathrm{OH}$ moiety of the catechol.

Interestingly, the oxidised groups in the above models did not disrupt cluster conjugation. However, surface oxidation can also result in basal plane disruption $[49,50]$, which we hypothesized could limit the ability to establish $\pi$-interactions between DA and the graphene surface. To confirm this, we modelled clusters displaying oxidised defects within the basal plane, namely a single epoxide and hydroxyl functionality, as shown in Figure $7 \mathrm{~d}$ and 7e, respectively. A weak attractive interaction was observed for DA at an epoxide site (DA-EP, Figure 7d); the DA molecule was found to be oriented further from the basal plane than DA-BP (3.4 $\AA$ ), and to interact with the oxygen of the epoxide through the - $\mathrm{OH}$ moiety 
a) $\mathrm{DA}-\mathrm{BP}$

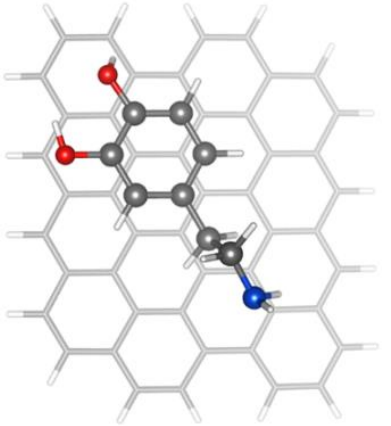

d) DA-EP
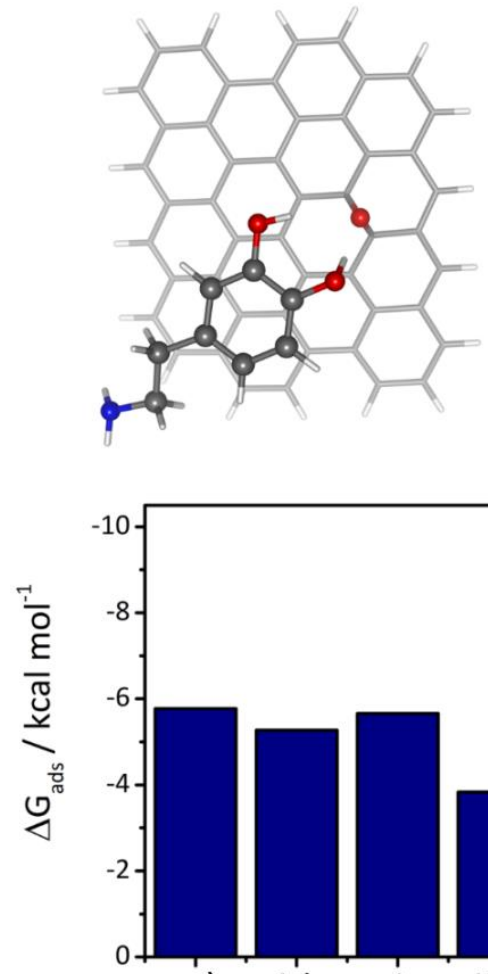

a) b) DA-CO

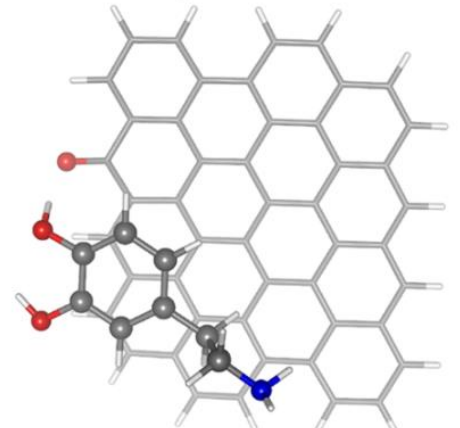

e) $\mathrm{DA}-\mathrm{OH}-\mathrm{B}$

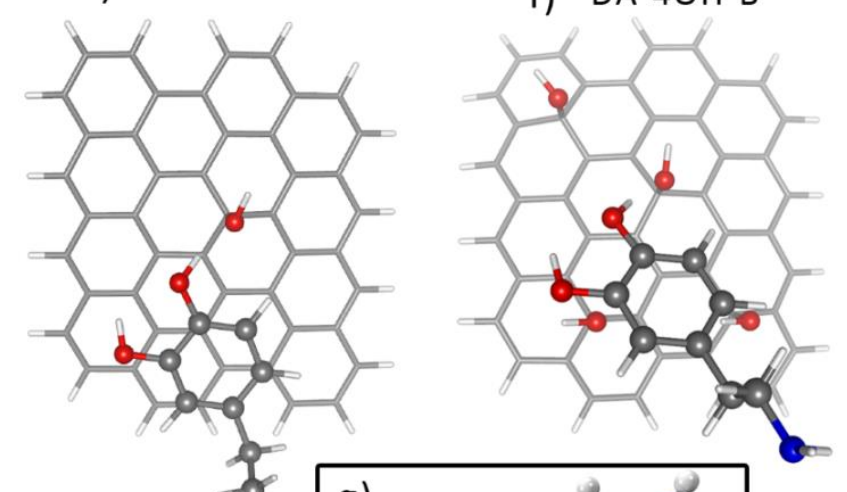

c) $\mathrm{DA}-\mathrm{OH}-\mathrm{E}$

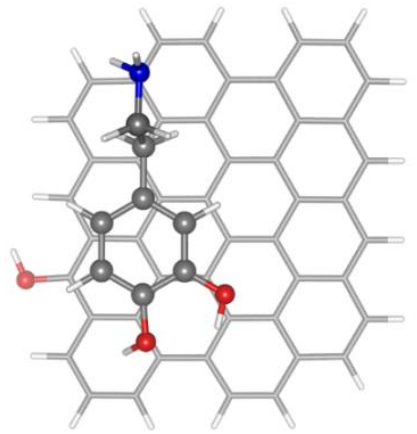

f) $\mathrm{DA}-4 \mathrm{OH}-\mathrm{B}$
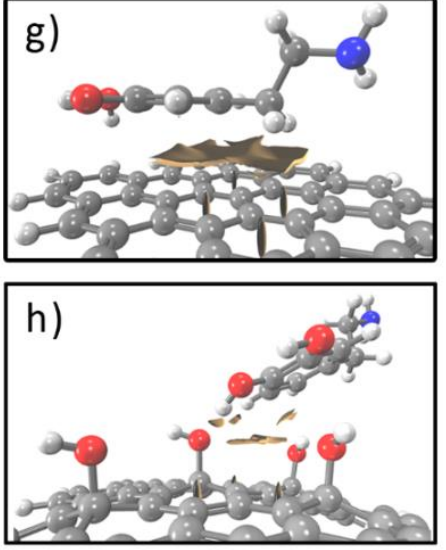

Figure 7. DA adsorption on model surfaces derived from DFT calculations. The corresponding structures for each $\Delta \mathrm{G}_{\mathrm{ads}}$ value are shown around the graph. a) DA-BP; b) DA-CO; c) DA-OH-E; d) DA-EP; e) DA-OH-B; and f) DA-4OH-B. g) and h) NCI plots (isovalue $=0.05$ a.u.) illustrating attractive van der Waals interactions for DA-BP and DA-4OH-B, respectively.

of the catechol (see Figure S9). In the case of DA interacting with a single hydroxyl group (DA-OH-B, Figure 7e), a significant decrease of $c a .4-5 \mathrm{kcal} \mathrm{mol}^{-1}$ in the $\Delta \mathrm{G}_{\mathrm{ads}}$ relative to DA-BP was observed. This enhancement stems from the ability of DA in DA-OH-B to establish attractive interactions both via the $\pi$-system and through $\mathrm{H}$-bonding with the $-\mathrm{OH}$ 
group (see Figure S10). This result strongly suggests that the presence of C-OH sites may not necessarily interfere with but could in fact enhance DA adsorption if interaction with the basal plane is not impeded.

The cluster in DA-OH-B represents an oxidised surface with a relatively low $\mathrm{OH}$ content; however, an increase in the density of hydroxyl (or other oxidised) groups may result in the disruption of $\pi$-interactions. Indeed, Figure $7 \mathrm{f}$ shows the result of a simulation for a cluster with $4 \mathrm{OH}$ surface groups (DA-4OH-B) in which the preferred orientation allows for favourable interactions, mostly through $\mathrm{H}$-bonding. This disruption of $\pi$-interactions can be more clearly visualised in the NCI plots presented in Figures $7 \mathrm{~g}$ and $7 \mathrm{~h}$, which show the attractive van der Waals interactions for DA-BP and DA-4OH-B, respectively. From these representations it is evident that the regions involved in the attractive $\pi$-interactions in DABP are more widespread and intense. The disruption of $\pi$-interactions caused by the increase in the $-\mathrm{OH}$ coverage is also reflected in a more positive $\Delta \mathrm{G}_{\mathrm{ads}}$, which highlights the importance of the surface coverage in DA adsorption, in agreement with our voltammetry studies as discussed below.

DA was found to adsorb on annealed GC which, based on the presence of a strong $\pi-\pi^{*}$ peak in the $\mathrm{C} 1 \mathrm{~s}$ spectrum, is expected to be comprised of large graphitic cluster regions. The presence of low amounts of oxidised groups, which are likely to be epoxides/hydroxyls based on the $\mathrm{O} 1 \mathrm{~s}$ spectrum, is not expected to significantly disrupt $\pi$-interactions between the aryl moiety of DA and the basal planes. In fact, O-groups might even promote adsorption if $\mathrm{H}$-bonding interactions can also be established at these sites. Anodised surfaces, despite their high density of oxidised groups, can also establish attractive interactions with DA; however, these are predominantly due to H-bonding with -OH groups of DA as suggested by DFT calculations. 
According to the $\Delta \mathrm{G}_{\mathrm{ads}}$ values presented in Figure $7, \mathrm{H}$ - and $\pi$-interactions in DA adsorbates appear to be comparable in strength, and when acting synergistically, they can result in the relatively strong binding of DA $\left(c a .-10 \mathrm{kcal} \mathrm{mol}^{-1}\right)$. However, we note that $\mathrm{H}$ bonding is expected to be dynamic in aqueous media, and therefore, it appears reasonable that highly oxidised surfaces cannot promote the formation of stable DA-adsorbates in aqueous electrolyte, in line with voltammetry results.

The saturation of existing oxide sites on the anodised surface with water molecules is therefore a plausible explanation for the absence of adsorbed DA. Nonetheless, transient attractive interactions on oxidised surfaces might still be possible via $\mathrm{H}$-bonding so that they can contribute to the catalysis of DA oxidation in the absence of a catalytic adsorbate layer. The rapid charge transfer kinetics observed on anodised GC cannot be explained by selfcatalysis through adsorbed DA, but it is possible that the large oxide coverage of the anodised surface is itself catalytic towards DA oxidation. The numerous oxide sites of the anodised surface may facilitate the approach of solution-phase DA without the need for pre-adsorption of the catechol at the electrode surface.

\section{Conclusions}

In this work we have studied the electrochemical response of model electrode surfaces prepared from glassy carbon electrodes in solutions of DA. Using a combination of voltammetry, X-ray photoelectron spectroscopy and DFT calculations, we have shown that charge transfer kinetics can be improved by pre-treatment of a polished glassy carbon surface using either an electrochemical oxidation or a thermal annealing treatment. Anodisation results in quasi-reversible charge transfer kinetics which, based on both the XPS spectra and computational studies, was attributed to catalysis of charge transfer through oxide moieties at the electrode surface. The annealed GC surface was found to have reversible charge transfer 
to DA despite having an identical oxygen content to polished GC, a feature which was attributed to the self-catalysis of charge transfer through an adsorbed layer of DA.

Based on the XPS studies of the annealed GC surface, the adsorption of DA was hypothesized to occur through non-covalent interactions between the $\pi$-system of the aryl ring of the catechol and graphitized regions of the surface. This hypothesis was supported by computational studies, which showed favourable values of $\Delta \mathrm{G}_{\mathrm{ads}}$ at the basal plane of graphene cluster surfaces. On the other hand, interruptions in the basal plane in the form of oxygen moieties result in hydrogen bonding interactions with the hydroxyl groups of the catechol ring. In the case of oxidation that still preserves regions of high graphitisation, stable DA-carbon adsorbates can still be formed. However, when $\pi$-interactions with the basal plane are significantly disrupted via oxidation, H-bonding is found to be the only means of establishing attractive interactions. Adsorption at such surfaces in aqueous media can only be transient due to competition from water as a far more abundant $\mathrm{H}$-bond donor, thus explaining why the highly oxidised surface of anodised GC displays no evidence of DA adsorption. However, H-bonding interactions between DA and -OH/epoxide groups are relatively strong, and might be sufficient to explain the fast charge transfer observed at anodized GC surfaces.

The interaction of DA with carbon surfaces is an important area of study due to the prevalence of functionalised carbon electrode materials in DA detection. This study clarifies the role of oxygen moieties and basal plane sites in facilitating both the adsorption of and charge transfer to DA at carbon electrode surfaces. The results are therefore expected to be of particular relevance to the electrochemical biosensor community. It is also likely that the conclusions reported herein may be generalised to the interaction of other catechol moieties or biologically-relevant aromatic compounds at carbon surfaces. Voltammetry studies with 
other catechols at carbon electrode surfaces are currently underway in our laboratory in order to test this hypothesis.

\section{Acknowledgements}

This publication has emanated from research conducted with the financial support of Science Foundation Ireland under Grant No. 13/CDA/2213 and of the Irish Research Council under Grant No. GOIPG/2014/399. D.R.J. acknowledges funding from the NSF of Sri Lanka under OSTP/2016/46. Use of the XPS of I. V. Shvets and C. McGuinness provided under SFI Equipment Infrastructure funds. We also acknowledge generous computing allocation time in the Kelvin cluster maintained by the Trinity Centre for High Performance Computing, which was funded through grants from the Higher Education Authority, through its PRTLI program.

\section{References}

[1] J.J. Schildkraut, The catecholamine hypothesis of affective disorders: a review of supporting evidence, Am. J. Psychiat., 122 (1965) 509-522.

[2] E. Dailly, F. Chenu, C.E. Renard, M. Bourin, Dopamine, depression and antidepressants, Fund. Clin. Pharmacol., 18 (2004) 601-607.

[3] O.D. Howes, S. Kapur, The Dopamine Hypothesis of Schizophrenia: Version III-The Final Common Pathway, Schizophrenia Bull., 35 (2009) 549-562.

[4] J. Lotharius, P. Brundin, Pathogenesis of parkinson's disease: dopamine, vesicles and $\alpha$ synuclein, Nat. Rev. Neurosci., 3 (2002) 932.

[5] M. Sofuoglu, R.A. Sewell, Norepinephrine and stimulant addiction, Addict. Biol., 14 (2009) 119-129. 
[6] K. Jackowska, P. Krysinski, New trends in the electrochemical sensing of dopamine, Anal. Bioanal. Chem., 405 (2013) 3753-3771.

[7] E. Forzani, G. Rivas, V. Solis, Amperometric determination of dopamine on an enzymatically modified carbon paste electrode, J. Electroanal. Chem., 382 (1995) 33-40.

[8] J. Njagi, M.M. Chernov, J. Leiter, S. Andreescu, Amperometric detection of dopamine in vivo with an enzyme based carbon fiber microbiosensor, Anal. Chem., 82 (2010) 989-996.

[9] Y.L. Zhou, R.H. Tian, J.F. Zhi, Amperometric biosensor based on tyrosinase immobilized on a boron-doped diamond electrode, Biosens. Bioelectron., 22 (2007) 822-828.

[10] J. Njagi, C. Ispas, S. Andreescu, Mixed Ceria-Based Metal Oxides Biosensor for Operation in Oxygen Restrictive Environments, Anal. Chem., 80 (2008) 7266-7274. [11] Z.-H. Sheng, X.-Q. Zheng, J.-Y. Xu, W.-J. Bao, F.-B. Wang, X.-H. Xia, Electrochemical sensor based on nitrogen doped graphene: Simultaneous determination of ascorbic acid, dopamine and uric acid, Biosens. Bioelectron., 34 (2012) 125-131.

[12] P. Gai, H. Zhang, Y. Zhang, W. Liu, G. Zhu, X. Zhang, J. Chen, Simultaneous electrochemical detection of ascorbic acid, dopamine and uric acid based on nitrogen doped porous carbon nanopolyhedra, J. Mater. Chem. B, 1 (2013) 2742-2749.

[13] E. Laviron, Electrochemical reactions with protonations at equilibrium: Part XII. The 2 $\mathrm{e}^{-}, 2 \mathrm{H}+$ homogeneous isotopic electron exchange reaction (nine-member square scheme), $\mathrm{J}$. Electroanal. Chem. Interfacial Electrochem., 169 (1984) 29-46.

[14] M.R. Deakin, P.M. Kovach, K. Stutts, R.M. Wightman, Heterogeneous mechanisms of the oxidation of catechols and ascorbic acid at carbon electrodes, Anal. Chem., 58 (1986) 1474-1480. 
[15] Q. Lin, Q. Li, C. Batchelor-McAuley, R.G. Compton, Two-Electron, Two-Proton Oxidation of Catechol: Kinetics and Apparent Catalysis, J. Phys. Chem. C, 119 (2015) 14891495.

[16] S. Corona-Avendaño, G. Alarcón-Angeles, M.T. Ramírez-Silva, G. Rosquete-Pina, M. Romero-Romo, M. Palomar-Pardavé, On the electrochemistry of dopamine in aqueous solution. Part I: The role of [SDS] on the voltammetric behavior of dopamine on a carbon paste electrode, J. Electroanal. Chem., 609 (2007) 17-26.

[17] C. Lin, L. Chen, E.E.L. Tanner, R.G. Compton, Electroanalytical study of dopamine oxidation on carbon electrodes: from the macro- to the micro-scale, Phys. Chem. Chem. Phys., 20 (2018) 148-157.

[18] S.H. DuVall, R.L. McCreery, Control of Catechol and Hydroquinone Electron-Transfer Kinetics on Native and Modified Glassy Carbon Electrodes, Anal. Chem., 71 (1999) 45944602 .

[19] M.R. Deakin, R.M. Wightman, The kinetics of some substituted catechol/o-quinone couples at a carbon paste electrode, J. Electroanal. Chem. Interfacial Electrochem., 206 (1986) 167-177.

[20] J.A. Behan, M.K. Hoque, S.N. Stamatin, T.S. Perova, L. Vilella-Arribas, M. GarcíaMelchor, P.E. Colavita, Experimental and Computational Study of Dopamine as an Electrochemical Probe of the Surface Nanostructure of Graphitized N-Doped Carbon, J. Phys. Chem. C, 122 (2018) 20763-20773.

[21] S.H. DuVall, R.L. McCreery, Self-catalysis by Catechols and Quinones during Heterogeneous Electron Transfer at Carbon Electrodes, J. Am. Chem. Soc., 122 (2000) 67596764.

[22] S. Kundu, Y. Wang, W. Xia, M. Muhler, Thermal Stability and Reducibility of OxygenContaining Functional Groups on Multiwalled Carbon Nanotube Surfaces: A Quantitative High-Resolution XPS and TPD/TPR Study, J. Phys. Chem. C, 112 (2008) 16869-16878. 
[23] J.-D. Chai, M. Head-Gordon, Long-range corrected hybrid density functionals with damped atom-atom dispersion corrections, Phys. Chem. Chem. Phys., 10 (2008) 6615-6620.

[24] M.J. Frisch, G.W. Trucks, H.B. Schlegel, G.E. Scuseria, M.A. Robb, J.R. Cheeseman, G. Scalmani, V. Barone, B. Mennucci, G.A. Petersson, H. Nakatsuji, M. Caricato, X. Li, H.P. Hratchian, A.F. Izmaylov, J. Bloino, G. Zheng, J.L. Sonnenberg, M. Hada, M. Ehara, K. Toyota, R. Fukuda, J. Hasegawa, M. Ishida, T. Nakajima, Y. Honda, O. Kitao, H. Nakai, T. Vreven, J.A. Montgomery, J.E. Peralta, F. Ogliaro, M. Bearpark, J.J. Heyd, E. Brothers, K.N. Kudin, V.N. Staroverov, R. Kobayashi, J. Normand, K. Raghavachari, A. Rendell, J.C. Burant, S.S. Iyengar, J. Tomasi, M. Cossi, N. Rega, J.M. Millam, M. Klene, J.E. Knox, J.B. Cross, V. Bakken, C. Adamo, J. Jaramillo, R. Gomperts, R.E. Stratmann, O. Yazyev, A.J. Austin, R. Cammi, C. Pomelli, J.W. Ochterski, R.L. Martin, K. Morokuma, V.G. Zakrzewski, G.A. Voth, P. Salvador, J.J. Dannenberg, S. Dapprich, A.D. Daniels, Farkas, J.B. Foresman, J.V. Ortiz, J. Cioslowski, D.J. Fox, Gaussian 09, Revision E.01, Gaussian Inc. , Wallingford CT, 2009.

[25] M.N. Penas-Defrutos, C. Bartolome, M. Garcia-Melchor, P. Espinet, Hidden arylexchange processes in stable 16e RhIII [RhCp*Ar2] complexes, and their unexpected transmetalation mechanism, Chem. Commun., 54 (2018) 984-987.

[26] E.R. Johnson, S. Keinan, P. Mori-Sánchez, J. Contreras-García, A.J. Cohen, W. Yang, Revealing Noncovalent Interactions, J. Am. Chem. Soc., 132 (2010) 6498-6506.

[27] J. Contreras-García, E.R. Johnson, S. Keinan, R. Chaudret, J.-P. Piquemal, D.N. Beratan, W. Yang, NCIPLOT: a program for plotting non-covalent interaction regions, J. Chem. Theory Comput., 7 (2011) 625-632.

[28] F.J. del Campo, P. Godignon, L. Aldous, E. Pausas, M. Sarrión, M. Zabala, R. Prehn, R.G. Compton, Fabrication of PPF Electrodes by a Rapid Thermal Process, J. Electrochem. Soc., 158 (2011) H63-H68. 
[29] R.G. Compton, C.E. Banks, Understanding Voltammetry, 2nd ed., Imperial College Press, London, 2011.

[30] R. Guidelli, G. Compton Richard, M. Feliu Juan, E. Gileadi, J. Lipkowski, W.

Schmickler, S. Trasatti, Defining the transfer coefficient in electrochemistry: An assessment (IUPAC Technical Report), Pure Appl. Chem., 2014, pp. 245.

[31] M. Cortés, H. Smida, C. Mériadec, F. Barrière, C. Lagrost, Direct SN1 reaction at oxidized PPF surfaces, Electrochem. Commun., 75 (2017) 48-51.

[32] G.K. Kiema, M. Aktay, M.T. McDermott, Preparation of reproducible glassy carbon electrodes by removal of polishing impurities, J. Electroanal. Chem., 540 (2003) 7-15.

[33] P. Takmakov, M.K. Zachek, R.B. Keithley, P.L. Walsh, C. Donley, G.S. McCarty, R.M. Wightman, Carbon Microelectrodes with a Renewable Surface, Anal. Chem., 82 (2010) 2020-2028.

[34] D.-Q. Huang, C. Chen, Y.-M. Wu, H. Zhang, L.-Q. Sheng, H.-J. Xu, Z.-D. Liu, The determination of dopamine using glassy carbon electrode pretreated by a simple electrochemical method, Int. J. Electrochem. Sci, 7 (2012) 5510-5520.

[35] A.A. Rabinca, M. Buleandra, A. Balan, I. Stamatin, A.A. Ciucu, Electrochemical Behaviour and Rapid Determination of L-Dopa at Electrochemically Pretreated Screen Printed Carbon Electrode, Electroanal., 27 (2015) 2275-2279.

[36] R.S. Nicholson, I. Shain, Theory of stationary electrode polarography. Single scan and cyclic methods applied to reversible, irreversible, and kinetic systems, Anal. Chem., 36 (1964) 706-723.

[37] A.J. Bard, L.R. Faulkner, J. Leddy, C.G. Zoski, Electrochemical Methods: Fundamentals and Applications, Wiley New York 1980. 
[38] E. Hershenhart, R.L. McCreery, R.D. Knight, In situ cleaning and activation of solid electrode surfaces by pulsed laser light, Anal. Chem., 56 (1984) 2256-2257.

[39] L. Chen, X. Li, E.E.L. Tanner, R.G. Compton, Catechol adsorption on graphene nanoplatelets: isotherm, flat to vertical phase transition and desorption kinetics, Chem. Sci., 8 (2017) 4771-4778.

[40] R.H. Wopschall, I. Shain, Effects of adsorption of electroactive species in stationary electrode polarography, Anal. Chem., 39 (1967) 1514-1527.

[41] E. Laviron, Adsorption, autoinhibition and autocatalysis in polarography and in linear potential sweep voltammetry, J. Electroanal. Chem. Interfacial Electrochem., 52 (1974) 355 393.

[42] C.D. Allred, R.L. McCreery, Adsorption of catechols on fractured glassy carbon electrode surfaces, Anal. Chem., 64 (1992) 444-448.

[43] N. Hellgren, R.T. Haasch, S. Schmidt, L. Hultman, I. Petrov, Interpretation of X-ray photoelectron spectra of carbon-nitride thin films: New insights from in situ XPS, Carbon, 108 (2016) 242-252.

[44] S. Yumitori, Correlation of C1s chemical state intensities with the O1s intensity in the XPS analysis of anodically oxidized glass-like carbon samples, J. Mater. Sci., 35 (2000) 139146.

[45] G.E. Cabaniss, A.A. Diamantis, W.R. Murphy Jr, R.W. Linton, T.J. Meyer, Electrocatalysis of proton-coupled electron-transfer reactions at glassy carbon electrodes, J. Am. Chem. Soc., 107 (1985) 1845-1853.

[46] U. Gelius, P.F. Hedén, J. Hedman, B.J. Lindberg, R. Manne, R. Nordberg, C. Nordling, K. Siegbahn, Molecular Spectroscopy by Means of ESCA III. Carbon compounds, Physica Scripta, 2 (1970) 70. 
[47] T. Okpalugo, P. Papakonstantinou, H. Murphy, J. McLaughlin, N. Brown, High resolution XPS characterization of chemical functionalised MWCNTs and SWCNTs, Carbon, 43 (2005) 153-161.

[48] H. Estrade-Szwarckopf, B. Rousseau, U.P.S. and X.P.S. studies of alkali-graphite intercalation compounds, Synth. Met., 23 (1988) 191-198.

[49] K. Erickson, R. Erni, Z. Lee, N. Alem, W. Gannett, A. Zettl, Determination of the Local Chemical Structure of Graphene Oxide and Reduced Graphene Oxide, Adv. Mater. (Weinheim, Ger.), 22 (2010) 4467-4472.

[50] K.N. Kudin, B. Ozbas, H.C. Schniepp, R.K. Prud'homme, I.A. Aksay, R. Car, Raman Spectra of Graphite Oxide and Functionalized Graphene Sheets, Nano Lett., 8 (2008) 36-41. 